Proceedings of the $2^{\text {nd }}$ ICEENG Conference, 23-25 Nov. 1999

\begin{tabular}{|l|l|}
\hline $\mathrm{AW}-1$ & 1 \\
\hline
\end{tabular}

$\begin{array}{ll}\begin{array}{l}\text { Military Technical College } \\ \text { Kobry Elkobbah, } \\ \text { Cairo, Egypt }\end{array} & \begin{array}{l}2^{\text {nd }} \text { International Conference } \\ \text { on Electrical Engineering }\end{array} \\ \text { ICEENG } 99\end{array}$

\title{
PLANE WAVE SCATTERING FROM PERIODIC ARRAYS ON GROUNDED CHIRAL SLAB
}

\author{
M. Medhat Mokhtar
}

\section{ABSTRACT}

Some scattering characteristics from a planar structure consists of a periodic array of patches on loss/lossless isotropic and reciprocal grounded chiral substrate are introduced. The results show that this structure has an absorption feature of the incident waves over a reasonable band of frequencies.

\section{KEY WORDS}

Microstrip antennas, Absorption material

* Egyptian Armed Forces 


\section{INTRODUCTION}

Electromagnetic chirality was experimentally emphasized at the beginning of the nineteenth century using a media composed of uniformly distributed and randomly oriented chiral objects like helix or irregular tetrahedron. Recent interests were motivated by many researches; e.g. remote sensing of certain types of terrestrial vegetation layers that behave as chiral media, and the scattering from and radiation through a plasma surrounding high speed moving objects, in addition to develop of new applications as chirowaveguides [1], and microstrip antennas [2]. Consequently it was necessary to develop the appropriate dyadic Green's function of the considered problem. It has been developed in the spatial domain for an unbounded media in a complex form [3], while that for planar structure is found more convenient for numerical computation in the spectral domain [4].

The purpose of the paper is to illustrate some scattering characteristics from a periodic array of patches on loss/lossiess isotropic and reciprocal grounded chiral substrate. The paper is organized as follows. In Section II, the main parameters and the constitutive relations for chiral material are briefly introduced to clarify the characteristics of the selected material. In Section Iil, some numerical results are presented to illustrate the effect of different material parameters on the scattered wave in comparison with the case of no chirality. The results show that the periodic arrays on grounded chiral slab has an absorption feature of electromagnetic waves over a reasonable band of frequencies. Finally the conclusion is presented in Section IV.

\section{PROBLEM FORMULATION}

The Maxwell's equations in the Chiral material are given by

$$
\nabla \times E=i \omega B, \text { and } \nabla \times H=-i \omega D
$$

with

$$
D=\varepsilon E+\gamma_{e} B, \text { and } H=\gamma_{m} E+B / \mu
$$

where $\varepsilon=\varepsilon_{o}\left(1+\chi_{e}\right)$ and $\mu=\mu_{o} /\left(1-\chi_{m}\right), \chi_{e}$ and $\chi_{m}$ are the electric and magnetic self susceptibilities, and $\gamma_{e}$ and $\gamma_{m}$ are the cross susceptibilities. In general, $\varepsilon, \mu, \gamma_{e}$ and $\gamma_{m}$ are complex quantities. The chiral media is lossless if $\varepsilon$ and $\mu$ are real, and $\gamma_{\theta}$ $=-\gamma_{m}$ and becomes reciprocal if $\gamma_{\mathrm{e}}=\gamma_{\mathrm{m}}=\mathrm{i} \gamma$ with real $\gamma$.

The solution of the wave equation, derived in a straight forward manner, provides two modes of circularly polarized wave with propagation wave numbers given by

$$
\begin{aligned}
& h_{1}=\omega \mu \gamma+\sqrt{(\omega \mu \gamma)^{2}+k^{2}} \text { for the right circularly polarized, and. } \\
& h_{2}=-\omega \mu \gamma+\sqrt{(\omega \mu \gamma)^{2}+k^{2}} \text { for the left circularly polarized, }
\end{aligned}
$$

where $k$ is the wave number in case of $\gamma=0$. Note that $h_{1}<k<h_{2}$ for $\gamma<0$ and $h_{2}<k<h_{1}$ for $\gamma>0$. 
At a boundary between a dielectric and chiral media, an incident plane wave propagating in the dielectric medium is splited into two circularly polarized propagating waves. The corresponding angles between both directions of propagation and the normal to the interface is given by

$$
\vartheta_{1}=\arcsin \left(\frac{k_{i} \sin \vartheta_{i}}{h_{1}}\right) \text {, and } \vartheta_{2}=\arcsin \left(\frac{k_{i} \sin \vartheta_{i}}{h_{2}}\right) \text {, }
$$

where $\vartheta_{i}$ is the angle of incidence, and $k_{i}$ is the wave number in the space. One of the two circularly polarized waves may propagate at the interface if the corresponding wave number is less than or equal $k_{i} \vartheta_{i}$. If both wave number fulfill this condition, total reflection of the incidence wave occurs.

Assume that a substrate of a chiral material is loaded by a perfectly conductor sheet at one side, and a uniform array of perfectly conductor patches at the dielectric/chiral boundary as shown in Fig.1. The scattered field due to a plane wave illumination is expressed as a doubly summing of an infinite number of propagating and evanesced modes that fulfill the boundary condition at the interface. The field of each mode is denoted by a subscript $(p, q)$ where $p$ and $q$ are the mode numbers in $x$ and $y$ directions respectively. Each propagating mode represents a plane wave propagating in a direction defined in terms of the array geometry. The scattered mode that verify the reflection or transmission law of the physical optic is the dominant mode $(0,0)$. The evanesced modes have an affect on the reflection characteristic curve associated with two types of resonance; the patch resonance and the array resonance $[5,6]$. In the following section, the reflection characteristic curves is defied by

$$
R=\frac{E_{(0,0)}^{s}+E^{r}}{E^{i}}
$$

where $E^{i}$ is the incident electric field, $E^{r}$ is the reflected electric field from the structure without the patch array, and $E_{(0,0)}^{s}$ is the dominate mode of the scattered field due to the induced surface current on the patches.

\section{NUMERICAL RESULTS}

Consider a problem of grounded dielectric substrate with $\varepsilon_{\mathrm{r}}=2.55$, and thickness $h=.0008 \mathrm{~m}$ loaded by a uniform square patch array of side measures $0.0025 \mathrm{~m}$ and equals half the periodicity length in both directions. Assume that the surface of the structure is illuminated by a linearly polarized plane wave with the electric field is parallel to one side of patches.

In Figs.2.a,b, and c, the reflection coefficient $|R|$, defined by Eqn. (4), versus the frequency is shown in the three cases of normal, $30^{\circ} \mathrm{TE}$, and $30^{\circ} \mathrm{TM}$ plane wave incidence, respectively. As discussed in [6], the higher order mode resonances depend on the patch size and normally occur at lower frequencies than that of the poles of the dyadic Green's function. Also, the dashed vertical line denotes the onset of the grating lobes in the space $(60 \mathrm{GHz}$ for normal incidence (NI) and $35.3 \mathrm{GHz}$ for $30^{\circ}$ oblique incidence). In the case of $\mathrm{NI}$, shown in Fig.2.a, the $|R|$ curve exhibits a wide dip in the frequency band beyond the $f_{G L}(60 \mathrm{GHz})$, while the presence of many 
higher order propagating modes have a negligible effect. Figs.2.b and c, show the $|R|$ curves for $30^{\circ} \mathrm{TE}$ and $30^{\circ} \mathrm{TM}$ incidence respectively. In both, there is a narrow dip followed by a wide one. In the case of $30^{\circ} \mathrm{TE}$, the narrow dip at $46 \mathrm{GHz}$ is related to the resonance of the $T E_{-1,0}$ mode, while the second case of $30^{\circ} \mathrm{TM}$ with the electric field in $\mathrm{x}$-direction produces a narrow dip near to $60 \mathrm{GHz}$ associated with the resonance of the $T E_{0, \pm 1}$ mode. Also, as in the case of $\mathrm{NI}$, the wide dip is a result of the grating lobe onset.

Assume that the dielectric substrate is replaced by a chiral one of the same thickness and permittivity with chirality admittance $\xi_{c}=0.005$. In this case, the $|R|$ has both the co-polar and cross-polar components, and the wave inside the substrate are left- and right-handed. Figs.3.a, b, and c, for the three cases of normal, $30^{\circ} \mathrm{TE}$, and $30^{\circ} \mathrm{TM}$ incidences respectively, show the absolute value of both the co-polar and cross-polar reflection coefficients, in addition to the total reflection coefficient $|R|$. In Fig.3.a, the case of $\mathrm{NI}$, there are shallow dips in the solid curve of the total reflected field in the frequency band below $\mathrm{f}_{\mathrm{GL}}$. with a narrow peak in the cross-polar curve at $57 \mathrm{GHz}$. Beyond $\mathrm{f}_{\mathrm{GL}}$, no wide dip can be noted as in the case in Fig.2.a (no chirality). The contribution of the higher order modes appears as two narrow adjacent dips. In the two cases of TE and TM oblique incidences, multi-narrow dips can be noted in Fig. 3.b, and c. It is worth noting that there is a deep dip in both Fig. 3.b and c similar to that in the cases of no chirality (see Figs.2.b and c); the first at $46 \mathrm{GHz}$ in the case of $30^{\circ} \mathrm{TE}$ due to the resonance of the $T E_{-1,0}$, while the second is around $60 \mathrm{GHz}$ in the case of $30^{\circ} \mathrm{TM}$ due to the resonance of the $\mathrm{TE}_{0, \pm 1}$. Obviously, the chirality of the material reduces significantly the level of the grating lobes.

The effect of increasing the thickness and the chirality admittance of the slab are depicted in Figs.4. $a$ and $b$ respectively for the case of NI. In comparison with Fig. 3.a, increasing either the thickness or the chirality admittance shifts down and broadens the frequency band of the higher order modes resonance.

Assume that the same array geometry is supported by a grounded loss chiral substrate of thickness $0.002 \mathrm{~m}$ and $\varepsilon_{\mathrm{r}}=1.35$. The reflection coefficient curve in the case of $\mathrm{NI}$ is shown in Figs.5.a, and $\mathrm{b}$ for the loss $\operatorname{tangent}(\tan \delta)=0.01$ and 0.1 respectively. In the two Figures, the thick solid line is the reflection coefficient curve of the supporting substrate without the array. The total, co-polar, and cross-polar reflection coefficient curves has a maximum value less than outlined by the thick solid line except in a very narrow band around $40 \mathrm{GHz}$. The main features of the curves in both Figures are very similar, and exhibit the effect of the higher order modes as dips at frequencies very close to that obtained in the case of lossless chiral substrate. The substrate with higher loss tangent produces more smooth reflection coefficient characteristics. It worth noting that a structure has a reflection characteristic curve as shown in Fig. 5.b is a good absorber over moderate range of frequencies. 
Proceedings of the $2^{\text {nd }}$ ICEENG Conference, 23-25 Nov. 1999

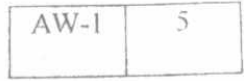

\section{CONCLUSION}

A composite material consists of uniformly distributed chiral elements immersed in a loss dielectric and covered by a periodic array of arbitrary-shaped perfectly conductor patches can be considered as an absorber for the electromagnetic waves over moderate range of frequencies. The proper selection of the structure geometry and parameters can produce a bounding surface for the higher order modes produced from the array., Multi-layer structure with different array geometries can be used for extending the absorption frequency band of interest.

\section{REFERENCES}

[1]D. Pozar, "Microstrip antennas and arrays on chiral substrates", IEEE Trans. Antennas Propagat. ,Vol.40, No.10, Oct. 1992, PP.1260-1263. [2]Pelet, P., and Engheta N., "The therory of chirowaveguides“, IEEE Trans. Antennas Propagat. ,Vol.38, No.1, 1990, PP.90-98. [3] Engheta, N., and Bassiri, S., "One- and two-dimentional dyadic Green's function in chiral media", IEEE Trans. Antennas Propagat., Vol.AP-37, No.4, Apr. 1989, PP. 512-515.

[4]Toscano A., and Vegni L., "Spectral dyadic Green's function formulation for plannar integrated structures with a grounded chiral slab", J. Electromagnetic Waves Applic., Vol.6, N0.5/6, 1992, PP.751-769.

[5] Ragab, A., Mokhtar, M., Allam, A., and Ibrahem, S., "Plane wave scattering from microstrip passive array", Submitted A.M.S.E. Journals.

[6] Allam, A., "Plane wave scattering from microstrip array of patches at arbitrary incidence", Proc. of ICEENG 98, March 1998, EGYPT.

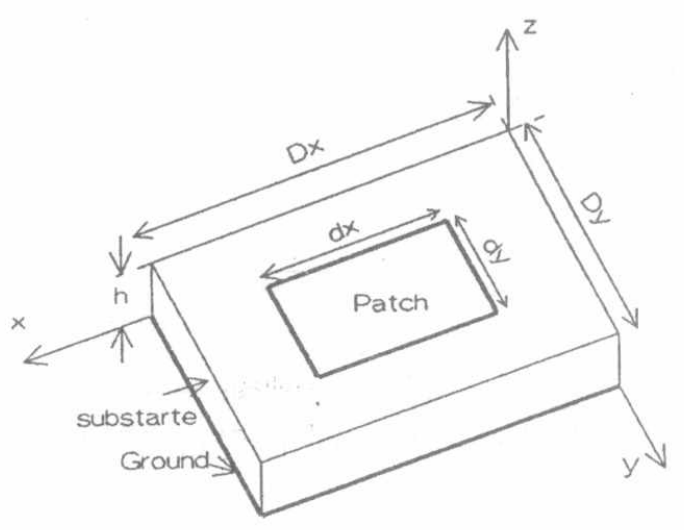

Fig.1 The geometry of a unit cell of the array 
Proceedings of the $2^{\text {nd }}$ ICEENG Conference, 23-25 Nov. 1999

\begin{tabular}{|l|l|}
\hline AW-1 & 6 \\
\hline
\end{tabular}
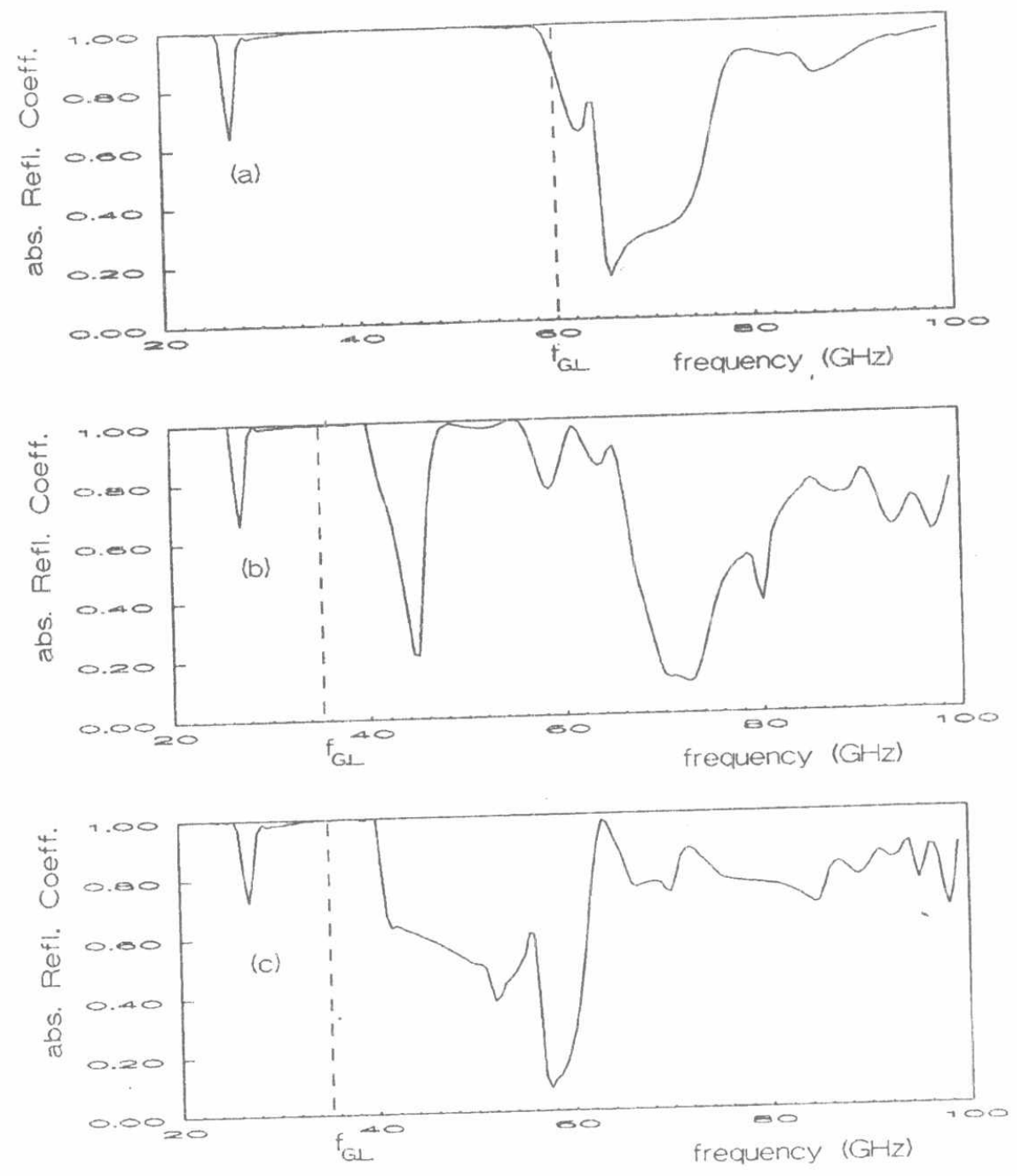

Fig.2 The R Vs. $f$ of. an array on grounded lossless dielectric substrate with $h=0.8 \mathrm{~mm}$; a)N Incid. b) $30^{\circ} \mathrm{TE}$ c/30 $\mathrm{TM}$ 

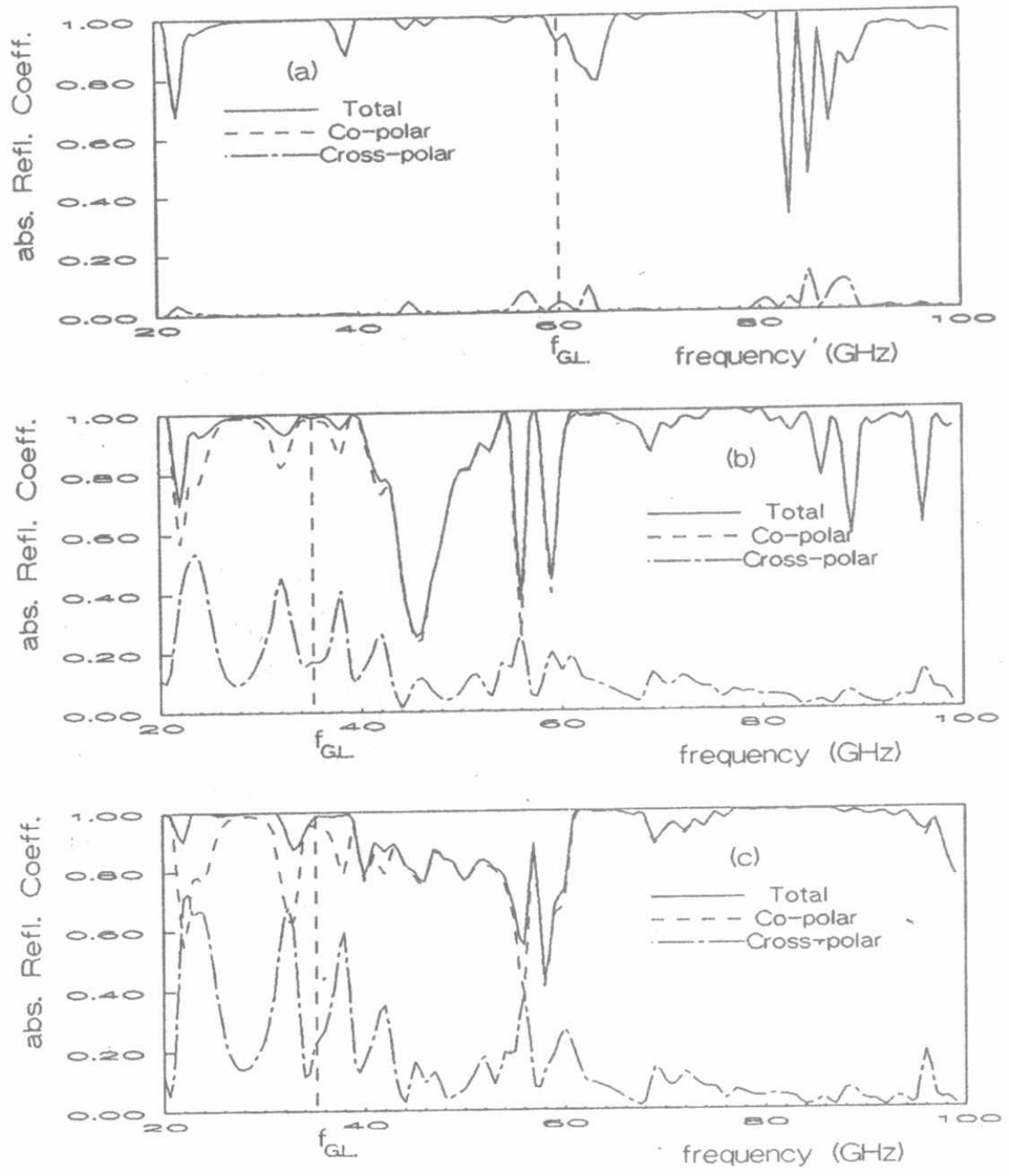

Fig.3 The R Vs. f. for the problem in Fig.2 but in case of Chiral substrate with $\xi_{c}=0.005$ 
Proceedings of the $2^{\text {nd }}$ ICEENG Conference, 23-25 Nov. 1999
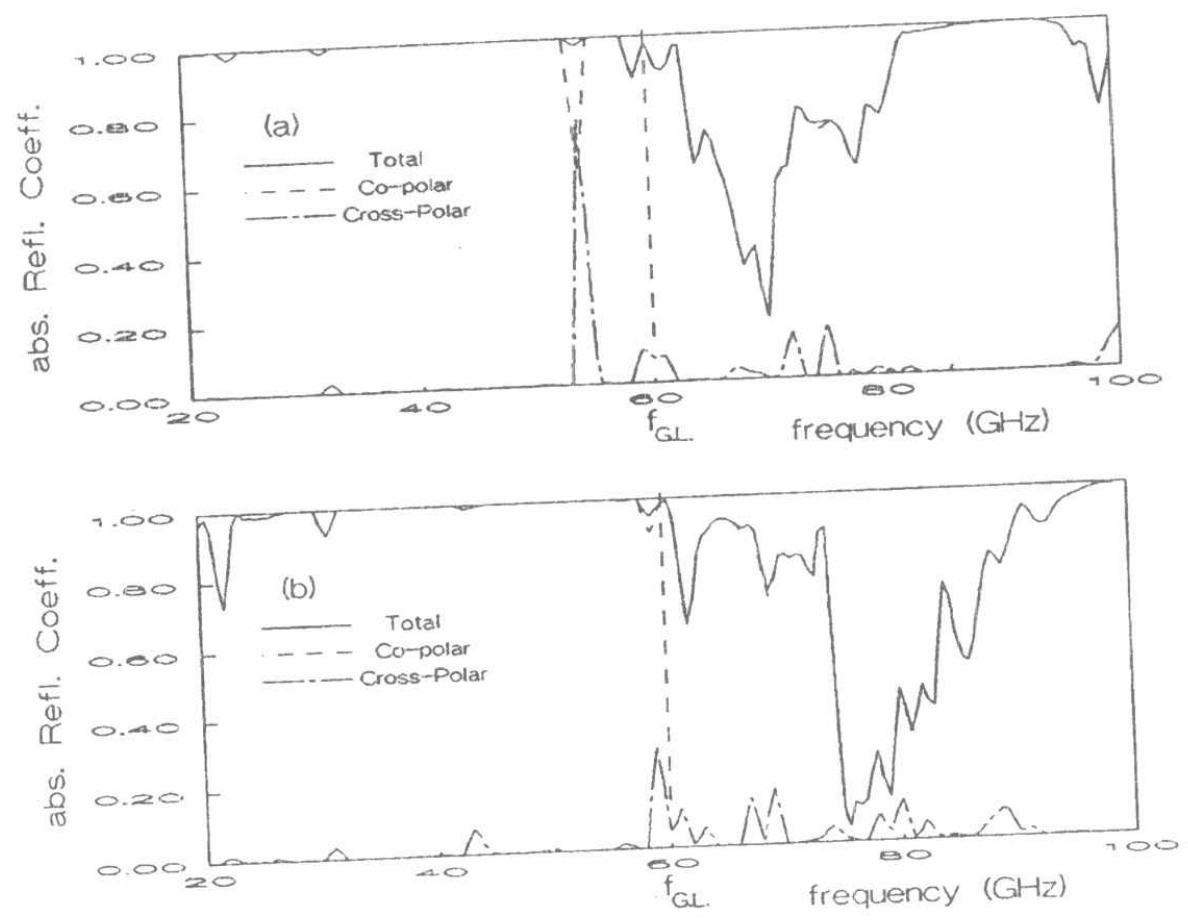

Fig.4 The A Vs. $f$ of an array on grounded lossless chiral substrate due to normal incidance illumination; a) $h=1.2 \mathrm{~mm}, \xi_{c}=0.005$. b) $h=0.8 \mathrm{~mm}, \xi_{c}=0.01$ 

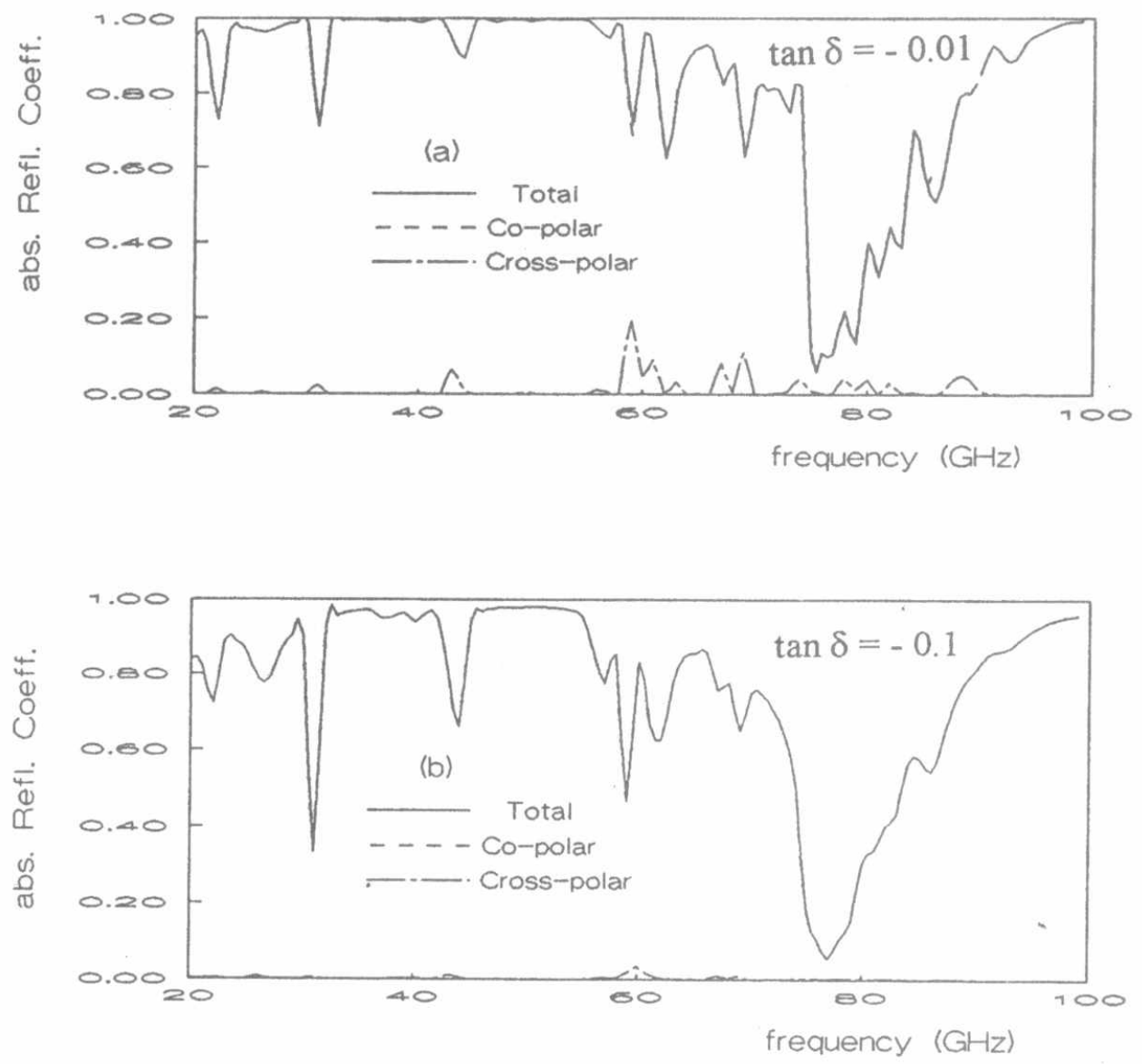

Fig.5 The R vs. $f$ of an array on grounded loss chiral substrate with $\xi_{\mathrm{c}}=0.005$ and $\mathrm{h}=1.2 \mathrm{~mm}$ due to $\mathrm{N}$. incidance illumination: 
